\title{
Estudo Comparativo dos Resultados Maternos e Perinatais entre Pacientes com Diabetes Pré-gestacional Tipo I e Tipo II
}

\author{
Comparative Study of Maternal and Perinatal Outcomes among Patients with \\ Pregestational Type I and Type II Diabetes
}

\begin{abstract}
Micheline Monte de Carvalho, Veruska Andrade de Mendonça, Carlos Augusto Alencar Júnior, Ana Paula Andrade Augusto, Marcelo Bezerra Nogueira
\end{abstract}

\section{RESUM0}

Objetivos: avaliar a evolução da gestação, controle metabólico e resultados perinatais de pacientes diabéticas pré-gestacionais e fazer um estudo comparativo entre os resultados de pacientes com diabetes insulino-dependente e não-insulino-dependente.

Métodos: análise retrospectiva de prontuários de 57 pacientes com diagnóstico de diabetes prégestacional que iniciaram pré-natal no Serviço de Medicina Materno-Fetal da MaternidadeEscola Assis Chateaubriand da Universidade Federal do Ceará, no periodo de janeiro 1995 a dezembro de 1998. As 57 grávidas incluidas no estudo foram divididas em dois grupos: grupo I, composto de 28 pacientes portadoras de diabetes insulino-dependente (tipo I), e grupo II, com 29 gestantes com diabetes não-insulino-dependente (tipo II) controladas com dieta ou com hipoglicemiante oral antes da gestação.

Resultados: não houve diferença estatisticamente significante entre os dois grupos em relação à necessidade de internamento para controle glicêmico $(39,2 \% \times 27,5 \%)$ e complicações maternas, tais como: hipertensão arterial crônica (14,2\% × 27,5\%), doença hipertensiva específica da gravidez $(14,2 \% \times 17,2 \%)$, amniorrexe prematura (3,5\% $\times 10,3 \%)$, infecção do trato urinário $(10,7 \% \times 6,8 \%)$ e trabalho de parto prematuro $(3,5 \% \times 6,8 \%)$. Foi observado, porém, maior número de episódios de hipoglicemia materna entre as pacientes insulino-dependentes (35,7\% $x 3,4 \%)$. Os resultados perinatais foram semelhantes. Observamos elevados indices de malformações e morbimortalidade perinatal.

Conclusão: não houve diferença na incidência de intercorrências clínicas e obstétricas entre as pacientes insulino-dependentes e não-insulino-dependentes, excluindo-se hipoglicemia materna.

PALAVRAS-CHAVE: Diabetes melito. Resultados perinatais. Complicações da gravidez. Malformações fetais.

\section{Introdução}

Diabetes mellitus é uma doença heterogênea que compreende desordens fisiopatológicas distintas no metabolismo dos carboidratos, decorrentes de uma complexa interação de fatores genéticos e ambientais ${ }^{1}$. As mulheres com dia-

Disciplina de Ginecologia e Obstetrícia

Departamento de Saúde Materno-Infantil

Maternidade - Escola Assis Chateaubriand

Universidade Federal do Ceará

Correspondência:

Carlos Augusto Alencar Júnior

Rua Dr. José Lourenço, 781 - apto. 1004 - Meireles

60115-280 - Fortaleza - CE betes na gravidez podem ser divididas em dois grupos: as com diabetes diagnosticado antes da gravidez (diabetes pré-gestacional) e aquelas com intolerância aos carboidratos diagnosticada durante a gestação (diabetes gestacional); esta última pode representar o primeiro sinal de diabetes franco.

As pacientes com diabetes pré-gestacional podem ou não depender do uso de insulina para se manterem compensadas, sendo desta forma classificadas em diabetes tipo I (insulino-dependente) e tipo II (não-insulino-dependente); ambas podem resultar em complicações tanto para a gestante como para o concepto ${ }^{2}$. 
Tem sido observada uma melhora significativa na evolução das gestações de mães diabéticas após a descoberta da insulina, com redução da incidência de cetoacidose, abortamentos espontâneos, natimortos e malformações congênitas. Observou-se queda da taxa de mortalidade perinatal de $65 \%$, antes da descoberta da insulina, para $2-5 \%$ nos dias atuais; e esta melhora dos resultados perinatais tem sido largamente atribuída ao bom controle glicêmico materno ${ }^{3-6}$.

Este trabalho tem por objetivo avaliar a evolução da gestação, controle metabólico e resultados perinatais de pacientes portadoras de diabetes pré-gestacional e fazer um comparativo entre os resultados de pacientes com diabetes insulinodependente e não-insulino-dependente.

\section{Pacientes e Métodos}

Foram analisados retrospectivamente os prontuários de 63 pacientes com diagnóstico de diabetes pré-gestacional que iniciaram pré-natal no Serviço de Medicina Materno-Fetal da Maternidade-Escola Assis Chateaubriand da Universidade Federal do Ceará, no período de janeiro de 1995 a dezembro de 1998. Foram excluídas seis pacientes porque seus partos ocorreram em outra maternidade ou por não terem completado o pré-natal em nosso serviço. As 57 grávidas restantes foram divididas em dois grupos para fins de estudo: o grupo I, com 28 pacientes portadoras de diabetes insulino-dependente (tipo I), e o grupo II, com 29 gestantes com diabetes nãoinsulino-dependente (tipo II) controladas com dieta ou hipoglicemiante oral antes da gestação. $O$ projeto foi e aprovado pela Comissão de Ética do Hospital.

Todas as pacientes incluídas no estudo fizeram acompanhamento pré-natal em nosso serviço até o final da gestação. A rotina de consultas foi semelhante nos dois grupos. Nas pacientes compensadas as avaliações foram realizadas mensalmente, no primeiro e segundo trimestres. No terceiro trimestre as consultas foram quinzenais e, após 34 semanas, semanais. As grávidas descompensadas foram mantidas internadas até o controle glicêmico e, posteriormente, seguiram rotina semanal ou quinzenal de consultas.

Os níveis glicêmicos foram controlados conjuntamente com o Serviço de Endocrinologia do Hospital Universitário Walter Cantídio durante todo o pré-natal por meio da dosagem da glicemia de jejum (normal $<105 \mathrm{mg} / \mathrm{dl}$ ) e pós-prandial (1 $1^{\text {a }}$ hora $<140 \mathrm{mg} / \mathrm{dl}$ e $2^{a}$ hora $<120 \mathrm{mg} / \mathrm{dl}$ ) e hemoglobina glicosilada (normal $<8,0 \%$ ).

Logo que iniciaram o pré-natal, as pacientes submeteram-se a estudo ultra-sonográfico, com a finalidade principal de determinar a normalidade e idade gestacionais. Repetiu-se o exame entre a $20^{a}$ e a $22^{\text {a }}$ semana, para avaliação da morfologia fetal, e entre a $28^{\text {a }}$ e a $34^{a}$ semana para se estudar o crescimento fetal, líquido amniótico e anexos. O exame foi repetido outras vezes conforme a necessidade de cada caso. Foram submetidas, também, a ecocardiograma fetal de rotina na $22^{\mathrm{a}}$ e na $26^{\underline{a}}$ semana para avaliação da anatomia cardíaca.

A vitalidade fetal foi analisada pela cardiotocografia e, quando esta se apresentava alterada, pelo perfil biofísico fetal. A dopplerfluxometria foi realizada apenas nas pacientes portadoras de vasculopatias, nas que desenvolviam doença hipertensiva específica da gravidez ou na suspeita de retardo de crescimento intra-uterino.

O tratamento do diabetes foi feito mediante de dieta com $35 \mathrm{cal} / \mathrm{kg} /$ dia fracionada em seis refeições, cada uma contendo $50 \%$ de carboidratos, $30 \%$ de lipídeos e $20 \%$ de proteínas, e quando necessário, terapia com insulina, utilizandose esquema combinado de insulina de ação intermediária (NPH) e insulina regular de forma também fracionada.

O momento do parto foi determinado de acordo com as condições maternofetais e, em alguns casos, foi realizada amniocentese para pesquisa de maturidade fetal pelo teste de Clements e contagem de células orangiófilas. Os recémnascidos foram avaliados logo após o nascimento pelo neonatologista e encaminhados à unidade de terapia intensiva quando indicado. Todos tinham suas glicemias avaliadas pelo menos durante as primeiras 24 horas de vida.

As gestantes foram caracterizadas pela idade, paridade e uso de insulina ou hipoglicemiante oral antes da gestação ou se faziam seu controle apenas com dieta. Foi determinada a idade gestacional de início do pré-natal e o controle glicêmico durante o mesmo, assim como as intercorrências maternas e fetais associadas. As seguintes complicações neonatais foram consideradas: hipoglicemia (glicemia $<40 \mathrm{mg} / \mathrm{dl}$ ), hiperbilirrubinemia (bilirrubina indireta $>12 \mathrm{mg} / \mathrm{dl}$ ), policitemia (hemoglobina $>22 \%$ ), hipocalcemia (cálcio $<7 \mathrm{mg} / \mathrm{dl}$ ), taquipnéia transitória do recémnascido, sindrome do desconforto respiratório, infecção, presença de malformação fetal e inadequação do peso ao nascer, sendo este considerado pequeno para a idade gestacional (PIG) quando o peso foi menor que o percentil 10, grande para a idade gestacional (GIG) quando maior que o percentil 90 e macrossômico quando o peso 
excedeu $4.000 \mathrm{~g}$.

Os dados foram inseridos no programa EpiInfo versão $6.0 \mathrm{e}$, para análise estatística, foram utilizados o teste exato de Fisher e o teste do $\chi^{2}$. $O$ valor do $\mathrm{p}<0,05$ foi considerado significante. Os dados relativos aos resultados perinatais foram analisados após exclusão dos abortamentos e óbitos fetais.

\section{Resultados}

Como referido anteriormente, foram comparadas as grávidas previamente diabética insulino-dependentes (DMID - grupo I) e nãoinsulino-dependentes (DMNID- grupo II).

A maioria das pacientes de ambos os grupos tinha idade entre $20-35$ anos $(71,4 \% \mathrm{x}$ $65,5 \%$, porém um número maior de grávidas apresentava idade superior a 35 anos no grupo II $(14,2 \%$ x $34,4 \%)$. Quanto à paridade, $71,4 \%$ do grupo I e $89,6 \%$ do grupo II eram multiparas e estas diferenças não foram estatisticamente significantes.

Entre as pacientes com DMID, 78,5\% não apresentavam nenhuma forma de vasculopatia (classes B e C de White) e apenas duas tinham retinopatia (classe $R$ ), uma delas com nefropatia associada (classe RF). No grupo II, 15 faziam uso de hipoglicemiante oral e 14 controlavam seus níveis glicêmicos apenas com dieta antes da gestação.

Infelizmente, a maioria das diabéticas iniciou o pré-natal tardiamente. Apenas $19,2 \%$ procuraram o serviço antes da $12^{\text {a }}$ semana, sendo portanto dificil avaliar se estavam compensadas no período da concepção. A média e o desvio padrão da idade gestacional de início do pré-natal foi $17,6 \pm 6,9$ semanas, no grupo I, e 18,6 $\pm 7,3$ semanas no grupo II.

Após exclusão das grávidas que abortaram, observamos que entre as pacientes com DMID, $76,9 \%$ necessitaram aumentar a dose de insulina durante a gestação, sendo a época média deste aumento $25,1 \pm 7,2$ semanas. A média da dose máxima necessária para controle glicêmico foi de 30 UI de insulina NPH e 13,8 UI de insulina regular. Nas com DMNID, metade necessitou utilizar insulina durante a gestação e o início da insulinoterapia se deu, em média, na 24,6 \pm 7,2 semana, porém a média da dose máxima para controle glicêmico foi menor que a necessária para as insulino-dependentes (16,7 UI de NPH e 5,6 UI de regular).

Não houve diferença estatisticamente significante entre os dois grupos em relação à necessidade de internação para controle glicêmico $(39,2 \% \times 27,5 \%)$ e complicações maternas, tais como: hipertensão arterial crônica $(14,2 \% \mathrm{x}$ $27,5 \%)$, doença hipertensiva específica da gravi$\operatorname{dez}(14,2 \% \times 17,2 \%)$, amniorrexe prematura $(3,5 \%$ x 10,3\%), infecção do trato urinário $(10,7 \%$ x 6,8\%) e trabalho de parto prematuro $(3,5 \% \times 6,8 \%)$ (Tabela 1). Foi observado, porém, maior número de episódios de hipoglicemia materna entre as pacientes insulino-dependentes $(35,7 \%$ × $3,4 \%)$, resultado estatisticamente significante, com $p=0,002$ e risco relativo de 10,3 , com intervalo de confiança grande $(1,4-75,6)$

Tabela 1 - Intercorrências clínicas e obstétricas nas pacientes com diabetes prégestacional insulino-dependente e não-insulino-dependente.

\begin{tabular}{|c|c|c|c|c|c|}
\hline & \multicolumn{2}{|c|}{ Tipo I $\mathrm{n}=\mathbf{2 8}$} & \multicolumn{2}{|c|}{ Tipo II $n=29$} & \multirow{2}{*}{$\begin{array}{c}\text { Significância } \\
\text { p }\end{array}$} \\
\hline & $\mathrm{n}$ & $\%$ & $\mathrm{n}$ & $\%$ & \\
\hline $\mathrm{HAC}$ & 4 & 14,2 & 8 & 27,5 & 0,22 \\
\hline DHEG & 4 & 14,2 & 5 & 17,2 & 0,89 \\
\hline Amniorrexe & 1 & 3,5 & 3 & 10,3 & 0,31 \\
\hline ITU & 3 & 10,7 & 2 & 6,8 & 0,87 \\
\hline TPP & 1 & 3,5 & 2 & 6,8 & 0,51 \\
\hline Hipoglicemia & 10 & 35,7 & 1 & 3,4 & $0,002^{*}$ \\
\hline Abortamento & 2 & 7,1 & 1 & 3,4 & 0,48 \\
\hline Polidrâmnio & 11 & 39,2 & 8 & 27,5 & 0,35 \\
\hline Sofrimento fetal & 5 & 17,8 & 6 & 20,6 & 0,78 \\
\hline Óbito fetal & 4 & 14,2 & 3 & 10,3 & 0,47 \\
\hline
\end{tabular}

* Estatisticamente significante. Teste do $\chi^{2}$

HAC - Hipertensão arterial crônica

DHEG - Doença hipertensiva específica da gravidez

ITU-Infecção do trato urinário

TPP-Trabalho de parto prematuro

Não houve diferença significante entre as taxas de abortamento $(7,1 \% \times 3,4 \%)$, sofrimento fetal agudo $(17,8 \%$ x $20,6 \%)$, óbito fetal $(14,2 \% \times$ $10,3 \%)$ e presença de polidrâmnio $(39,2 \% \mathrm{x}$ $27,5 \%)$. Dos 7 óbitos fetais, 4 foram do grupo I, detectados com 35 e 36 semanas e um deles associado com prolapso de cordão, e 3 foram do grupo II, com idade gestacional de 35 a 37 semanas. Nenhum dos natimortos era portador de anomalias congênitas.

O tempo médio de resolução da gestação foi de 36,6 semanas $( \pm 2,4)$ para as pacientes com DMID e 37,6 semanas $( \pm 1,9)$ nas com DMNID. A prematuridade ocorreu em $50,0 \%$ das pacientes do grupo I e em $36,0 \%$ do grupo II (Tabela 2), porém três pacientes do grupo I tiveram seus partos entre 28-33 semanas, o que não ocorreu com nenhuma paciente do grupo II, em que todos os prematuros nasceram entre 34-36 semanas. 
Dentre as causas de prematuridade tivemos amniorrexe prematura, trabalho de parto prematuro, sofrimento fetal agudo e doença hipertensiva específica da gravidez em sua forma grave.

Tabela 2 - Resultados neonatais perinatais em gestantes diabéticas, previamente à gravidez, insulino-dependentes e não-insulino-dependentes.

\begin{tabular}{|c|c|c|c|c|c|}
\hline & \multicolumn{2}{|c|}{ Tipo I $\mathrm{n}=22$} & \multicolumn{2}{|c|}{ Tipo II $n=25$} & \multirow{2}{*}{$\begin{array}{c}\text { Significância } \\
\text { p }\end{array}$} \\
\hline & $\mathrm{n}$ & $\%$ & $\mathrm{n}$ & $\%$ & \\
\hline Hipoglicemia & 8 & 36,3 & 6 & 24,0 & 0,35 \\
\hline Hiperbilirrubinemia & 8 & 36,3 & 7 & 28,0 & 0,54 \\
\hline Malformação & 3 & 13,6 & 2 & 8,0 & 0,44 \\
\hline TTRN & 5 & 22,7 & 10 & 40,0 & 0,20 \\
\hline $\mathrm{DMH}$ & 2 & 9,0 & 4 & 16,0 & 0,39 \\
\hline Infecção & 2 & 9,0 & 3 & 12,0 & 0,56 \\
\hline Plaquetopenia & 1 & 4,5 & 1 & 4,0 & 0,72 \\
\hline Feto GIG & 10 & 45,4 & 9 & 36,0 & 0,12 \\
\hline Macrossomia & 8 & 36,3 & 6 & 24,0 & 0,35 \\
\hline Anoxia & 2 & 9,0 & 3 & 12,0 & 0,85 \\
\hline Óbito neonatal & 2 & 9,0 & 0 & 0,0 & 0,21 \\
\hline
\end{tabular}

TTRN - Taquipnéia transitória do recém-nascido

DMH-Doença da membrana hialina

GIG-Grande para a idade gestacional

Excluindo-se os abortamentos, verificamos que a taxa de cesárea foi de $80,7 \%$ no grupo I e $64,2 \%$ no II, sendo a indicação mais freqüente, em ambos os grupos, a macrossomia fetal (6 e 5 casos), seguida por sofrimento fetal agudo (4 e 5 casos).

Não foi observada diferença estatisticamente significante entre os resultados perinatais dos dois grupos, como visto na Tabela 2. Dentre as malformações encontradas tivemos a trissomia do 21 , craniossinostose, pé torto congênito, comunicação intra-atrial e um recém-nascido polimalformado. Em relação ao peso ao nascer, $45,4 \%$ dos neonatos do grupo I e $24,0 \%$ dos do grupo II foram macrossômicos.

Entre os 47 recém-nascidos vivos tivemos dois óbitos (4,2\%), ambos no grupo I, um por infecção neonatal e outro decorrente de múltiplas malformações.

\section{Discussão}

Nosso estudo teve como objetivo principal comparar os resultados perinatais entre gestantes com diabetes insulino-dependente e não- insulino-dependente prévio à gestação, além de avaliar o controle glicêmico durante o pré-natal e a evolução da gestação.

Em relação à idade, foi observado que a maioria das gestantes de ambos os grupos se encontravam no intervalo entre 20 e 35 anos. Observou-se, porém, que o grupo II teve maior prevalência de mulheres em idade avançada ( $>35$ anos), provavelmente devido ao fato de o DMNID ter incremento de sua incidência nessa faixa etária, mas esta diferença não foi significante. Sacks et al. $^{7}$, que realizaram estudo semelhante, evidenciaram diferença significante entre as diabéticas tipos I e II não só em relação à idade, mas também quanto à paridade, raça, índice de massa corpórea pré-gestacional e ganho de peso na gestação.

O bom controle glicêmico na época da concepção e nas primeiras semanas de gestação é de suma importância na prevenção de malformações fetais em pacientes diabéticas ${ }^{8}$. A dosagem da hemoglobina glicosilada no 1o trimestre é o método mais utilizado para avaliação deste risco $^{5,9}$. O controle metabólico no início da gestação também está relacionado com os resultados perinatais, como foi relatado por Mello et al. ${ }^{10}$, que estudaram 31 gestantes portadoras de DMID e verificaram niveis glicêmicos no $1^{\circ}$ trimestre mais baixos entre as que tiveram bons resultados perinatais. Nossas pacientes, entretanto, iniciaram tardiamente o pré-natal, não podendo ser avaliadas quanto ao controle glicêmico no período da concepção.

Como esperado, devido ao aumento da resistência periférica à insulina que ocorre na gestação a partir do $2^{\circ}$ trimestre, $76,9 \%$ das pacientes insulino-dependentes necessitaram aumentar a dose de insulina em média na 25aㅡ semana. Entre as diabéticas tipo II metade precisou usar insulina, com idade gestacional média no início do uso de 24,5 semanas. A média da dose máxima para controle glicêmico foi menor no grupo II, explicável pela maior deficiência pancreática nas diabéticas tipo I.

A necessidade de internação para controle glicêmico não foi diferente entre os grupos, mas foram observados mais episódios de hipoglicemia materna entre pacientes com diabetes insulinodependente $(35,7 \% \times 3,4 \%, p=0,002)$, com intervalo de confiança grande $(1,4-75-6)$, provavelmente devido ao número reduzido de gestantes. A incidência de hipoglicemia entre gestantes com diabetes tipo I situou-se entre 19 e $71 \%$, sendo mais freqüente durante o sono e naquelas com história prévia de hipoglicemia grave ${ }^{2}$. Sacks et al. ${ }^{7}$ observaram também maior incidência de hipoglicemia nas pacientes com DMID (19\%) em 
comparação com as portadoras de DMNID (2\%).

Os abortamentos espontâneos são mais freqüentes em diabéticas com controle glicêmico inadequado pré-gestacional, com risco três vezes maior. Pacientes com excelente controle têm risco equivalente às não diabéticas ${ }^{3,4,7}$. Nossa taxa de abortamento espontâneo foi de $7,1 \%$ nas pacientes com DMID, comparável aos $7,2 \%$ relatados por Hanson et al. ${ }^{11}$ e menor que os $14,3 \%$ registrados por Nordström et al. ${ }^{3}$. Talvez nosso baixo percentual seja decorrente do início tardio do prénatal, impedindo que gestantes que abortaram precocemente fossem incluidas na pesquisa.

A mortalidade fetal é quase duas vezes mais alta entre diabéticas que nas não-diabéticas $(10,4 \% \times 5,7 \%)^{4}$. A causa deste aumento ainda não foi determinada, mas observou-se ser mais freqüente após a 36 a semana e na presença de doença vascular, pior controle glicêmico, préeclâmpsia, polidrâmnio e macrossomia ${ }^{4,5}$. Nossa taxa foi de 14,2\% para as diabéticas tipo I e 10,3\% nas do tipo II. Ambas foram mais altas que as encontradas por Sacks et al. ${ }^{7}$, que não relataram óbito fetal entre 46 gestantes insulino-dependentes e taxa de 1,7\% entre as não-insulino-dependentes com idade superior a 35 anos.

Observamos taxas de cesáreas de 80,7\% no grupo I e $64,2 \%$ no II, maiores que as relatadas na literatura, que variam de 45 a $59 \%$ para as insulino-dependentes e são de $40 \%$ para as nãoinsulino-dependentes. A indicação mais freqüente, em ambos os grupo, é a macrossomia seguida do sofrimento fetal agudo ${ }^{3,5,12}$. Acreditamos que nosso elevado número de partos abdominais seja devido à dificuldade de avaliação intraparto da vitalidade fetal e ao dificil controle glicêmico das gestantes.

Uma das complicações neonatais mais freqüentes em gestantes diabéticas é o aumento do peso fetal, podendo a macrossomia acometer de 15 a $45 \%$ dos neonatos ${ }^{4}$. O controle glicêmico inadequado está associado ao aumento do risco para macrossomia. Entretanto, mesmo com controle excelente as diabéticas ainda têm um risco maior quando comparadas às não-diabéticas. Isso é explicado, provavelmente, pela influência de outros fatores sobre o crescimento fetal, tais como, multiparidade, obesidade materna pré-gestacional, aumento dos triglicérides, fatores genéticos, idade materna e niveis séricos do cordão de IGF$\mathrm{I}^{4,8,9,13}$. Entre as pacientes do nosso trabalho, no grupo I (insulino-dependentes) 45,4\% dos recémnascidos apresentavam peso acima do percentil 90 para a idade gestacional e taxa de macrossomia de $36,3 \%$, maior que a observada por
Nordström et al. ${ }^{3}$, que relatam que apenas $18 \%$ dos neonatos tinham peso maior que o percentil $90^{3}$. Landon et al. ${ }^{14}$ observaram 9\% de macrossomia em pacientes com DMID bem controlados, resultados bem inferiores aos 34\% das que tiveram controle inadequado. Já Stenninger et al. ${ }^{12}$ detectaram taxa de macrossomia de 48\% em 29 gestantes com DMID. No grupo II, 36,6\% dos neonatos eram grandes para a idade gestacional, com taxa de macrossomia de $24,0 \%$. Sacks et al. ${ }^{7}$ observaram proporção similar de recém-nascidos grandes para a idade gestacional em gestantes com DMNID (34\%).

A hipoglicemia neonatal é intercorrência comum em infantes de mães diabéticas, especialmente naquelas com controle glicêmico inadequado, entre eles a incidência é de até 50\%. Na nossa casuística, o quadro esteve presente em $36,3 \%$ dos recém-nascidos do grupo I, superior aos percentuais costumeiramente referidos, que variam de $3,2 \%$ a $25 \% \%^{3,7,10}$. Entre as pacientes com DMNID, 6 crianças apresentaram hipoglicemia. A diferença entre os dois grupos não teve significância estatística, como é relatado na literatura ${ }^{7}$.

Outra importante complicação neonatal é a sindrome do desconforto respiratório, cuja incidência ao longo dos últimos anos tem sido reduzida a cerca de $3 \%$. A causa precisa da maior incidência em filhos de mães diabéticas é desconhecida. Vários fatores vêm sendo implicados: hiperinsulinemia, hiperglicemia, níveis altos de mioinositol ${ }^{4,5}$. Esta sindrome foi diagnosticada em 9,0\% dos neonatos do grupo I e, de forma inexplicada, em $16 \%$ dos do grupo II.

As malformações congênitas ocorrem em 2$3 \%$ dos recém-nascidos da população geral, com aumento de até oito vezes entre filhos de mães diabéticas. Dentre as causas, a mais conhecida é a hiperglicemia, porém outros fatores teratogênicos vêm sendo implicados: os corpos cetônicos, radicais livres, inibidores da somatomedina, hipoglicemia, excesso de beta-hidroxibutirato, deficiência de vitaminas A e E, zinco, mioinositol e ácido araquidônico. Estes fatores, quando presentes antes da 7ํㅗ semana de gestação, podem levar a anomalias cardiacas e defeitos abertos do tubo neural, dentre outras, sendo a malformação mais característica a sindrome da regressão caudal. O controle glicêmico no período pré-concepcional e $1^{\circ}$ trimestre é, portanto, de suma importância na prevenção de anomalias congênitas, levando a taxas de malformações semelhantes às de mães não-diabéticas $^{2,4,5,7,9,13,15,16}$.

Nossos resultados revelaram taxa de 13,6\% de neonatos malformados em mães com DMID e 
de $8 \%$ nas com DMNID semelhantes às de outros estudos nos quais a incidência variou de 4,2 a $11,9 \%$ nas com DMID e de 2,7 a $14,1 \%$ nas com DMNID $^{11,15}$. Acreditamos que nossos elevados percentuais denotem o pobre controle glicêmico no início da gestação.

Este estudo inclui limitado número de pacientes devido à baixa prevalência do diabetes melito pré-gestacional. Foi possivel observar, entretanto, que não houve diferença na incidência de intercorrências entre as pacientes insulinodependentes e não-insulino-dependentes, excluindo-se a hipoglicemia materna. Apesar de os resultados maternos serem aparentemente satisfatórios, os dados perinatais foram extremamente preocupantes. Tivemos elevados indices de malformações, de mortalidade perinatal da prematuridade, além da acentuada morbidade neonatal. Estes dados refletem, provavelmente, a dificuldade que tivemos de obter um controle glicêmico apropriado. Reconhecemos as limitações que tivemos, em uma população socioeconomicamente desfavorecida, para o acompanhamento do diabetes. A dificuldade econômica, sem dúvida, influenciou nossos resultados. A impossibilidade de retornos mais freqüentes ou de monitorização domiciliar da glicemia são exemplos de como o fator financeiro pode interferir no resultado do binômio mãe-filho nesta difícil desordem endocrinológica.

Além disso, nossas pacientes iniciaram tardiamente o pré-natal, não podendo ser avaliadas quanto ao controle glicêmico no período da concepção. O início tardio do pré-natal é fator preocupante em pacientes sabidamente diabéticas antes da gravidez. A falta de acesso aos serviços pré-natais, seja por culpa da paciente ou do próprio sistema de saúde, poderia ser fator arrolado como causador do fato. Preocupante, também, é a ausência de esclarecimento às diabéticas pelos clínicos que as acompanham, propiciando demora no primeiro atendimento durante a gravidez.

Fica claro, por fim, que a presença do diabetes previamente à gestação, independente do tipo, e não obstante todos os avanços propedêuticos e terapêuticos atualmente existentes, continua sendo patologia preocupante a denotar cuidados extremos por parte do obstetra e de toda a equipe multidisciplinar que acompanha a grávida.

\section{SUMMARY}

Purpose: to evaluate the evolution of gestation, metabolic control and perinatal outcome of pregestational diabetic patients and to perform a comparative study of the results of patients with insulin-dependent diabetes (type I) and noninsulin-dependent diabetes (type II).

Methods: retrospective analysis of 57 pregestational diabetic woman charts who began a prenatal follow-up in the Service of Maternofetal Medicine of the MaternidadeEscola Assis Chateaubriand of the Universidade Federal do Ceará, in the period from January 1995 to December 1998. The 57 pregnant women included in the study were divided into groups: the first, composed of 28 patients with insulin-dependent diabetes (type I), and the second with 29 pregnant women with non-insulin-dependent diabetes (type II), controlled with diet or with oral hypoglycemics before pregnancy.

Results: there was no statistically significant difference between the two groups in relation to the need of hospitalization for glycemia control (39.2\% x 27.5\%) and maternal complications, such as: chronic arterial hypertension $(14.2 \% \times 27.5 \%)$, pregnancy-induced hypertension $(14.2 \% \times 17.2 \%)$, premature rupture of membranes $(3.5 \% \times 10.3 \%)$, urinary tract infection $(10.7 \%$ $x$ 6.8\%), and preterm labor (3.5\% x 6.8\%). However, episodes of maternal hypoglycemia were more frequent among insulin-dependent patients (35.7\% x 3.4\%). The perinatal results were similar. We observed a great number of congenital anomalies and increased perinatal morbidity and mortality.

Conclusion: there was no difference in the incidence of obstetric and clinical complications between insulindependent and non-insulin-dependent patients, except for maternal hypoglycemia.

KEY WORDS: Diabetes mellitus. Perinatal outcome. Pregnancy complications. Fetal malformation.

\section{Referências}

1. Sacks DB. Implications of the revised criteria for diagnosis and classification of diabetes mellitus. Clin Chem 1997; 43:2230-2.

2. Linn T, Bretzel RG. Diabetes in pregnancy. Eur J Obstet Gynecol Reprod Biol 1997; 75:37-41.

3. Nordström L, Spetz E, Wallstöm K, Walinder O. Metabolic control and pregnancy outcome among women with insulin-dependent diabetes mellitus. A twelve-year follow-up in the country of Jämtland, Sweden. Acta Obstet Gynecol Scand 1998; 77:284-9. 
4. Moore TR. Diabetes in pregnancy. In: Creasy RK, Resnik R, editores. Maternal Fetal Medicine: principles and practice. $4^{\text {th }}$ ed. Philadelphia: W.B. Saunders; 1999. p.964-95.

5. Landon MB. Diabetes mellitus and other endocrine diseases. In: Gabbe SG, Niebyl JR, Simpson JL, editores. Obstetrics: normal and problem pregnancies. $3^{\text {rd }}$ ed. New York: Churchill Livingstone; 1996. p.1037-60.

6. Rudge MVC, Calderon IMP, Ramos MD, Maestá I, Souza LMS, Peraçoli JC. Perspectiva perinatal decorrente do rígido controle pré-natal em gestações complicadas pelo diabete. Rev Bras Ginecol Obstet 1995; 17:26-32.

7. Sacks DA, Chen W, Greenspoon JS, Wolde-Tsadik G. Should the same glucose values be targeted for type 1 as for type 2 diabetics in pregnancy? Am J Obstet Gynecol 1997; 177: 1113-9.

8. Gold AE, Reilly R, Little J, Walker JD. The effect of glycemic control in the pre-conception period and early pregnancy on birth weight in women with IDDM. Diabetes Care 1998; 21:535-8.

9. Mironiuk M, Kietlinska Z, Jezierska-Kasprzyk K, Piekosz-Orzechowska B. A class of diabetes in mother, glycemic control in early pregnancy and occurrence of congenital malformations in newborn infants. Clin Exp Obstet Gynecol 1997; 24:193-7.
10.Mello G, Parretti E, Mecacci F, Pratesi M, Lucchetti R, Scarselli G. Excursion of daily glucose profiles in pregnant women with IDDM: relationship with perinatal outcome. J Perinat Med 1997; 25:488-97.

11.Hanson U, Persson B, Thunell S. Relationship between haemoglobin A1C in early type 1 (insulin-dependent) diabetic pregnancy and the occurrence of spontaneous abortion and fetal malfomation in Sweden. Diabetologia 1990; 33:100-4.

12.Stenninger E, Schollin J, Aman J. Early postnatal hypoglycaemia in newborn infants of diabetic mothers. Acta Paediatr 1997; 86:1374-6.

13.Johnstone FD. Pregnancy management in women with insulin-dependent diabetes. Br J Hosp Med 1997; 58:207-10.

14.Landon MB, Gabbe SG, Piana R, Mennuti MT, Main EK. Neonatal morbidity in pregnancy complicated by diabetes mellitus: predictive value of maternal glycemic profiles. Am J Obstet Gynecol 1987; 156:1089-95.

15.Lowy C. Outcome of pregnancy in women with insulin-dependent diabetes. Rate of congenital malfomations is almost certainly gross underestimated. BMJ 1998; 316:550-2.

16. Mauad Filho F, Dias CC, Meirelles RS, Cunha SP, Nogueira A, Duarte G. Diabetes e gravidez: aspectos clínicos e perinatais. Rev Bras Ginecol Obstet 1998; 20:193-8.

\section{COMUNICADO AOS ASSOCIADOS E LEITORES}

\section{FEBRASGO na Internet}

Para informações e consultas temos à disposição quatro endereços eletrônicos: 